\title{
1 Truncation of IFT80 causes early embryonic loss in cattle
}

2

3 M. Sofía Ortega*, Derek M. Bickhart*, Kelsey N. Clark, Daniel J. Null, Jana L. Hutchison,

4 Jennifer C. McClure, John B. Cole ${ }^{\dagger}$

5

$6 *=$ co-first author

$7 \quad \dagger=$ corresponding author

8

$9{ }^{1}$ Cell Wall Biology and Utilization Research Laboratory, U.S. Dairy Forage Research Center,

10 Agricultural Research Service, United States Department of Agriculture, Madison, WI, 53706

11

12 2Division of Animal Sciences, College of Agriculture, Food, and Natural Resources, University

13 of Missouri, Columbia, 65211

14

$15{ }^{3}$ Animal Genomics and Improvement Laboratory, Henry A. Wallace Beltsville Agricultural

16 Research Center, Agricultural Research Service, United States Department of Agriculture,

17 Beltsville, MD, 20705-2350 
ABSTRACT

Recessive alleles represent a substantial risk in populations that have undergone significant bottleneck events. Analyzing millions of genotypes and hundreds of sequenced bulls, we

21 identified one pernicious recessive variant that achieved 1.66\% frequency in the US Holstein

22 population and confirmed loss of embryonic development via CRISPR-Cas9 editing. In the

23 process, we identified the developmental significance of two protein domains of the IFT80 gene

24 in the embryonic development of cattle.

\section{MAIN}

26 Known lethal recessive alleles account for substantial economic losses to cattle breeders ${ }^{1}$, and 20

27 such defects ${ }^{2}$ are routinely tracked in the US population of 9.4 million dairy cows. Economic

28 losses resulting from embryonic death and stillbirths caused by these alleles have been estimated

29 to cost farmers at least $\$ 11$ million per year ${ }^{1}$ in the US and approximately $£ 1.8$ million annually

30 in the UK. The advent of artificial insemination techniques has exacerbated the problem by

31 increasing the frequency of recessive lethal alleles in commercial herds. The HH2 allele, which

32 accounts for annual fertility losses of more than \$2 million in the US alone, is present in $1.66 \%$

33 of the domestic Holstein population ${ }^{3}$ and has a significant effect on cow fertility in the form of

34 early embryonic losses. The patrilineal nature of cattle reproduction and the availability of large

35 numbers of genotyped animals provides an opportunity to identify and validate such recessive

36 mutations and measure their potential impacts on other species.

38 We first sought to refine the $\mathrm{HH} 2$ genomic locus to narrow the window for variant discovery in 39 subsequent sequencing analysis (Fig 1a). Our analysis was aided by the recent release of a high40 quality reference assembly for taurine cattle ${ }^{4}$ which had over 100 -fold more contiguity than the 
41 previously used UMD3.1 reference ${ }^{5}$. Using imputed SNP genotypes ${ }^{6}$ derived from more than 3.7

42 million SNP genotypes of commercial cattle mapped to the new reference assembly, we were

43 able to reduce the size of the locus from $8.9 \mathrm{Mbp}$ to $1.5 \mathrm{Mbp}$. This reduced the number of

44 candidate genes that were previously hypothesized to be causal for the lethality of the haplotype

45 by $72 \%$, leaving only seven genes in the refined window out of 25 original candidates.

47 Separate, non-overlapping datasets were used for causal variant discovery and validation. The

48 discovery dataset included whole-genome sequence data for 9 carriers and 449 non-carriers from

49 the Animal Genomics and Improvement Laboratory (Beltsville, MD), the Collaborative Dairy

50 DNA Repository (Madison, WI) and the 1000 Bull Genomes Project ${ }^{7}$. A concordance analysis

51 was performed in order to identify candidate causal variants within the haplotype locus, and one

52 INDEL (Fig 1b) that was $>99 \%$ concordant with known carrier status was identified. One carrier

53 animal was predicted to not have a heterozygous copy of the INDEL, but manual review of read

54 pileups on the variant site identified the INDEL at a frequency of $25 \%$ which was below the

55 threshold of initial detection (data not shown). We interpreted this result as complete

56 concordance and attributed the lower proportion of variant reads to a bias in sampling the variant

57 haplotype during sequencing library preparation. The concordant INDEL was located on

58 btau1:107,172,615 and was found to induce a frameshift in the IFT80 gene's 11th exon that led

59 to early truncation of the protein product (Supplementary Figure 1). In silico translation of the

60 expected protein product of the edited transcript suggested an early truncation of the IFT80

61 protein, resulting in a protein of 385 amino acids (49.5\% of total) compared to the wild type.

62 The early termination of the gene interrupts one of seven WD 40-repeat containing domains

63 (Pfam: SSF50978) and a predicted weak polyampholyte domain 
64 (https://mobidb.bio.unipd.it/A0A4W2EVR7), both of which likely impact the intra-flagellar transport of materials in the cell ${ }^{8}$. Analysis of potential founders identified the bull Willowholme Mark Anthony (73HO0219, born 1975) as a likely candidate. Pedigree, SNP and DNA sequence

67 data show that he is the founder with respect to the SNP haplotype, but he is not a carrier of the actual IFT80 variant, which appears to have arisen in one of his daughters (Elysa Anthony Lea,

HOCANF000003628269, born 1981) or grand-daughters (Comestar Laurie Sheik-ET,

71 is not available, so the true founder cannot be identified.

73 We hypothesized that the early termination of the IFT80 gene was the causal variant of the

74 phenotype given its perfect concordance and the potential for the mutation to have a high impact

75 on downstream protein function. To induce the phenotype, we used a CRISPR-Cas9 gene editing

76 system (see Methods) to induce double-strand breaks at different exons at or upstream of the

77 candidate causal variant site (Fig 2a). In the first model, IFT80 was truncated at exon 2, and in a

78 second model, IFT80 was truncated at exon 11 (variant site). In both cases, treated embryos

79 failed to progress past the 8- to 16-cell transition period (Fig 2b, 2c; P $<0.0001$ ), and

80 degenerated after this developmental stage. As alternative splicing could potentially recover

81 IFT80 protein function, we quantified the expression at different exons of IFT80 transcript. For

82 embryos edited at exon 2, expression was measured at exons 1, 3-4 and 5-7 (before and after

83 edit site; Fig 2d). For the embryos edited at exon 11 (exon with causal mutation), IFT80

84 expression was determined at exons 9-10 and 12 (before and after the edited exon; Fig 2e) by

85 qPCR assays. Upstream regions of edited sites had no difference in IFT80 expression (Exon 1, P

$86=0.44$; Exon 9-10, $\mathrm{P}=0.62$ ), between edited and non-edited embryos. Conversely, in both 
87 models (truncation at exon 2 or 11) downstream exons of edited site, had significantly lower IFT80 expression $(\mathrm{P}<0.0001)$ in edited embryos than non-edited embryos, suggesting early

89 termination of the transcript due to the mutation or the altered splicing of these exons from the

90 transcript (Fig 2d). Differential expression was most pronounced in exon 12, in which the edited

91 cell line had $62.5 \%$ lower fold expression of the exon than the wildtype.

92

93 Mutations to the IFT80 gene are linked to asphyxiating thoracic dystrophy 2 (ATD2) disease in

94 human $^{9}$ and hypomorphic expression of the gene has been shown to result in low postnatal

95 survival in gene-trap mice ${ }^{10}$. Previous surveys have speculated as to when IFT80 gene expression

96 is essential during development ${ }^{10}$, and our results suggest that expression is needed to progress

97 past the 8- to 16-cell developmental phase. This is consistent with previous RNA surveys of

98 cattle preimplantation embryonic development ${ }^{11}$, and with the known fertility effects of HH2 in

99 cattle $^{1,3}$. The results of editing experiments suggest that the carboxy-terminal WD 40-repeat or

100 the polyampholyte domains are essential for embryonic development. Recent studies suggest that

101 deletion of IFT80 disrupt FGF2 signaling pathway, affecting cell proliferation, and self-renewal

102 of odontoblasts ${ }^{12}$. In the preimplantation mammalian embryo, FGF2 signaling is essential for

103 lineage commitment and blastocyst formation ${ }^{13,14}$, in which disruption of FGF2 signaling

104 coincides with the developmental arrest observed in this study.

106 Using genotypes from millions of Holstein cattle and CRISPR-Cas9 gene editing, we were able

107 to track and validate a recessive lethal allele in the IFT80 gene, respectively. This approach

108 conclusively identified the target mutation in such a way that it provided further insight into the

109 function of the gene in development of other mammalian species. There is a further economic 
benefit to this discovery, as identification of the exact causal mutation underlying this haplotype

111 will benefit dairy producers by allowing them to avoid carrier-to-carrier matings which result in

112 pregnancy losses responsible for $\sim \$ 437,000 /$ year in US Holsteins. Concordance analysis and

113 molecular validation on naturally occurring genetic variants represent a potent tool for ongoing

114 efforts to translate genotype to phenotype in the field of Quantitative Genetics.

\section{ONLINE METHODS}

\section{Haplotype discovery}

117 Possible autosomal recessive mutations are routinely tracked during the imputation step of the 118 national genomic evaluations calculated by the Council on Dairy Cattle Breeding (CDCB;

Bowie, MD). Genotypes from 47 different arrays are currently stored in the CDCB database, with SNP counts ranging from 2,900 to 777,962, and are imputed to a set of 79,276 (80k) for

121 genomic evaluation. No single chip includes all these markers, regardless of density, and

122 genomic calculations require that genotypes are available for all 80k SNP. Chromosomes are

123 phased into maternal and paternal haplotypes and the genome is divided into 100-SNP segments.

124 Inheritance of those segments is traced through the population and missing SNP are filled in

125 using a segment from the pedigree when available, and using the most common segment in the

126 population when pedigree information is not available ${ }^{3}$. Most males have complete pedigrees

127 available for many generations, but many females do not. For this study, haplotyping was

128 performed using the program Findhap version $3^{15}$. Haplotypes that either never appear in the

129 homozygous state, or which appear with far lower frequency than expected, are flagged as

130 possible recessives ${ }^{3}$. Such haplotypes are then tested for effects on fertility and stillbirth.

131 Founder animals are identified from the pedigree when possible. Currently, 26 such conditions

132 are tracked in the U.S. dairy cattle population 
(https://www.ars.usda.gov/ARSUserFiles/80420530/Publications/ARR/Haplotype tests_ARR-

134 Genomic5.pdf), and causal mutations are known for all but the HH2 haplotype.

In April of 2011 a haplotype affecting fertility was identified on Bos taurus autosome (BTA) 1 in

137 the region of 92-97 Mbp and designated as HH2 (Figure 1a). At that time, a second haplotype on

138 BTA 1 at 101-106 Mbp with a similar carrier list, lack of homozygotes and negative effects on

139 fertility also was identified. When a new SNP list was introduced in August 2011 the haplotype

140 in the 101-106 Mbp region was no longer flagged as a potential lethal. A new 61,000 SNP list

141 was introduced in December 2013, and the haplotype segment upstream from HH2 again

142 appeared on the list of possible lethal haplotypes. In April 2020 the location of HH2 was

143 changed to correspond with the upstream segment based on results from fine-mapping described

144 below. Haplotype blocks are formed using fixed numbers of SNP, rather than LD boundaries, so

145 it can sometimes be difficult to narrow the candidates down to a single haplotype. In this case,

146 both segments appear to track the causal variant with high concordance.

147 Whole-genome sequencing, variant calling, and annotation

148 The dataset used for causal variant discovery and validation included whole-genome sequence

149 data for 9 carriers and 763 non-carriers, including animals from the Ayrshire, Holstein, and

150 Jersey breeds sequenced by the Animal Genomics and Improvement Laboratory (Beltsville,

151 MD), the Collaborative Dairy DNA Repository (Madison, WI), and the 1000 Bull Genomes

152 Project ${ }^{7}$. Sequencing depth for these animals averaged 14.7x. Sequence data were aligned to the

153 ARS-UCD1.2 reference genome ${ }^{4}$ using BWA-MEM as implemented in bwa version 0.7.15-

$154 \mathrm{r} 1140^{16}$ and converted to the binary Sequence Alignment/Map format by samtools version $1.3^{17}$.

1551000 Bull Genomes run8 data were processed using version 4 of the Genome Analysis Toolkit ${ }^{18}$, 
and included genotyping, variant recalibration for SNP and INDEL, removal of individuals that

157 failed QC, and filtering of monomorphic alleles. The resulting VCF file was filtered to remove

158 reads with QS $<10$ and those identified as "LOWQUAL". Sequence data variants were

159 identified using the Samtools v1.9 and BCFtools v1.919 and reads passing edits were aligned

160 against version ARS-UCD1.2 of the bovine genome ${ }^{4}$. The list of filtered variants was annotated

161 with the SNPEff ${ }^{20}$ utility using the Ensembl annotation of the cattle reference genome (version

162 101). Variants listed as "High", "Medium" and "Low" priority within the haplotype region by

163 SNPEff were selected for further analysis. Impacts on predicted protein translation were

164 identified using custom scripts.

165 Production of IFT80-/- embryos

166 Guide RNA design

167 Guide RNAs (gRNAs) were designed to target exon 2 (Supplementary Table 1), or exon 11

168 (Supplementary Table 2) using the GPP portal from the Broad Institute

169 (https://portals.broadinstitute.org/gpp/public/analysis-tools/sgrna-design). To select gRNAs, two

170 criteria were used: (1) those with the highest on-target score after design based on the GPP portal

171 and the guide RNA checker portal (IDT technologies, San José, CA), and (2) those having the

172 least similarity with other bovine sequences containing a protospacer adjacent motif (PAM).

173 Selected gRNAs, as well as the universal 67mer tracrRNA® for form guide complexes were

174 ordered from IDT technologies.

175

176 The gRNAs and tracrRNA were resuspended to a concentration of $500 \mathrm{ng} / \mu \mathrm{l}$. Each guide was

177 then annealed with equal amounts of tracrRNA by heating at $95^{\circ} \mathrm{C}$ for 5 min and then cooled

178 down from 95 to $25^{\circ} \mathrm{C}$ using a gradient of $-1{ }^{\circ} \mathrm{C} / 12 \mathrm{~s}$ in a Veriti 96 -well gradient thermal cycler 
179 (ThermoFisher, CA) to obtain a concentration of $250 \mathrm{ng} / \mu 1 \mathrm{gRNA}-$ tracrRNA complex. Prior to zygote injection, gRNA-tracrRNA complexes were annealed to CAS9 mRNA (IDT

181 Technologies) by incubation at room temperature for $15 \mathrm{~min}$. The final injection solution was 50 ng/ $\mu \mathrm{l}$ each gRNAs complex and $20 \mathrm{ng} / \mu \mathrm{l}$ Cas 9 mRNA. For each exon targeted, guides were

183 injected in pairs, to create a sizeable cut in the sequence and facilitate rapid genotyping on an

184 agarose gel. The expected cuts for guides targeting exon 2 was 82 bp and for those targeting

exon 11 were expected to generate a cut of $331 \mathrm{bp}$.

\section{Production of embryos in vitro and zygote microinjection}

187 Embryos were produced in vitro with a single sire known to be of high fertility in vitro as

188 previously described ${ }^{21}$. At the end of fertilization, putative zygotes (oocytes exposed to sperm)

189 were denuded from the surrounding cumulus cells and split in two groups: injected only with

190 CAS9 mRNA and injected with gRNA/CAS9 mRNA against IFT80. During the injection

191 procedure zygotes were maintained manipulation medium ${ }^{22}$, and then transferred to four-well

192 dishes in groups of up to 50 zygotes in $500 \mu 1$ of SOF-BE2 ${ }^{23}$, covered with $300 \mu 1$ of mineral oil

193 per well at $38.5^{\circ} \mathrm{C}$ in a humidified atmosphere of $5 \%(\mathrm{v} / \mathrm{v}) \mathrm{O}_{2}$ and $5 \%(\mathrm{v} / \mathrm{v}) \mathrm{CO}_{2}$. Percentage of

194 putative zygotes that cleaved was determined at day 3 of development (day $0=$ day of

195 insemination) and blastocyst rate was estimated at day 8 of development. In addition, to

196 determine any developmental arrest, cell stage of all embryos was recorded at days 3, 5 and 8 of

197 development (Supplementary Figure 2).

\section{Editing efficiency}

199 To determine editing efficiency of the guides, embryos subjected to microinjection were

200 collected individually at day 8 of culture, placed in $6 \mu \mathrm{L}$ of Embryo Lysis Buffer ${ }^{22}$, and 
201 subjected to $30 \mathrm{~min}$ at $60^{\circ} \mathrm{C}$, followed by $10 \mathrm{~min}$ at $85^{\circ} \mathrm{C}$ in a Veriti 96 -well gradient thermal

202 cycler (ThermoFisher, CA). Lysed embryo solution was used as template DNA.

203 For genotyping, the region surrounding the target sequenced of the gRNAs was amplified by

204 endpoint PCR using the following primers $\left(5^{\prime} \rightarrow 3^{\prime}\right)$ : exon 2 F-

205

GGTTTCTTATCCTGCTTTCCATTC; R- GAAATTGAGTGTGAACCTTGGG, and for Exon

207 products were 639- and 620 bp for exon 2 (Supplementary Figure 3) and 11 (Supplementary

208 Figure 4), respectively. The PCR reaction, amplification (annealing temperature $53.4{ }^{\circ} \mathrm{C}$ ), and

209 visualization of bands was performed as previously described ${ }^{22}$. Editing efficiency was

210 calculated from 3 separate in vitro embryo production runs, with 100 embryos per treatment

211 group per replicate. Editing efficiency for exon 2 was $26 \%$ bi-allelic, $32 \%$ mono-allelic, and

$21242 \%$ non-edited, and for exon 11 was 50\% bi-allelic, 23\% mono-allelic, and 27\% non-edited.

\section{Nucleic acids isolation and RT-qPCR expression analysis}

214 DNA and RNA were isolated from individual 8-cell embryos (65-72 h post insemination)

215 injected against IFT80 or injected only with CAS9 mRNA. Embryos were collected and lysed

216 individually in $25 \mu \mathrm{l}$ of RLT Lysis buffer (Qiagen, Valencia, CA), lightly vortexed, transferred

217 to a nano column (PuroSPINTM Luna NANOTECH, Toronto, ON, CA), and centrifuged for $30 \mathrm{~s}$

218 (all centrifugations were performed at 16,000 g). At this point, DNA is trapped in the column

219 and the flow-through contained the RNA. The column was placed in a new collection tube and

220 stored at room temp for later DNA purification. The flow-through containing RNA was mixed

221 with one volume of $70 \%$ ethanol, transferred to a new nano column, and centrifuged for $30 \mathrm{~s}$.

222 Immediately after, $600 \mu 1$ of buffer RW1 (Qiagen) were added to the column, and centrifuged for

22330 s. Flow-through was discarded from the collection tube and $500 \mu 1$ of buffer RPE (Qiagen) 
224 were added to the column and centrifuged for $30 \mathrm{~s}$. After discarding the flow-through, $500 \mu \mathrm{l}$ of

$22580 \%$ ethanol were added to the column, and centrifuged for $2 \mathrm{~min}$. To elute RNA, the column

226 was placed in a new microcentrifuge tube, and $15 \mu l$ of RNAse-free water were added directly to

227 the membrane of the column, incubated at room temperature for one minute and finally

228 centrifuged for $1 \mathrm{~min}$. To complete DNA isolation, $500 \mu \mathrm{l}$ of $70 \%$ ethanol were added to the

229 nano column containing DNA, followed by a 1-min centrifugation. Then, the column was placed

230 in a new microcentrifuge tube and $15 \mu \mathrm{l}$ of EB buffer (Qiagen) preheated at $70{ }^{\circ} \mathrm{C}$ were added

231 directly to the membrane of the column, incubated at room temperature for $10 \mathrm{~min}$, followed by

2321 min centrifugation to elute DNA. Embryos edit status was first assessed using PCR across the

233 site of the edit as described above, and the RNA from homozygous edited embryo cells were

234 selected for further analysis. Due to the small amount of total RNA in each cell, homozygous

235 embryos were pooled in groups of four prior to cDNA creation. Three pools of edited and control

236 embryos were analyzed in subsequent analyses. Two strand cDNA synthesis was conducted

237 using the high-capacity cDNA Reverse transcription kit (ThermoFisher) reverse transcriptase

238 following manufacturer instructions. Primers sequences for each locus were designed using the

239 PrimerQuest ${ }^{\mathrm{TM}}$ (IDT technologies). All primers were validated following procedures previously

240 described ${ }^{24}$. QPCR was conducted using the CFX384 Touch Real-Time System (Bio-Rad) using

$241 \quad 10 \mu 1$ of reaction mix as previously described ${ }^{22}$. The gene $S D H A$ was chosen as a suitable

242 housekeeping gene for relative expression calculation in bovine embryos ${ }^{25}$. Fold changes were

243 calculated relative to the housekeeping gene $\left(2^{\Delta \mathrm{CT}}\right)$ as previously described ${ }^{22,24}$.

244 Statistics

245 Differences in gene expression and development were analyzed by least-squares analysis of

246 variance of the $\triangle \mathrm{CT}$ values using the GLM procedure of the Statistical Analysis System version 
2479.4 (SAS Institute Inc., Cary, NC, USA). Replicate and treatment were included as main effects

248 in the model.

\section{Acknowledgments}

250 Bickhart was supported by appropriated projects 8042-31000-001-00-D, "Enhancing Genetic

251 Merit of Ruminants Through Improved Genome Assembly, Annotation, and Selection” and

252 5090-31000-026-00-D, “Investigating Microbial, Digestive, and Animal Factors to Increase

253 Dairy Cow Performance and Nutrient Use Efficiency”, of the Agricultural Research Service

254 (ARS) of the United States Department of Agriculture (USDA). Cole, Hutchison and Null were

255 supported by appropriated project 8042-31000-002-00-D, “Improving Dairy Animals by

256 Increasing Accuracy of Genomic Prediction, Evaluating New Traits, and Redefining Selection

257 Goals", of ARS, USDA. McClure was supported by appropriated project 5090-31000-026-00-D,

258 "Investigating Microbial, Digestive, and Animal Factors to Increase Dairy Cow Performance and

259 Nutrient Use Efficiency" of ARS, USDA. Clark was supported by USDA NIFA Grant 2019-

$260 \quad 38420-28972$.

261

262 The authors thank US dairy producers for providing phenotypic, genomic, and pedigree data

263 through the Council on Dairy Cattle Breeding under USDA Agricultural Research Service (ARS)

264 Material Transfer Research Agreement 58-8042-8-007. Access to whole-genome sequence data

265 from the Collaborative Dairy DNA Repository (Madison, WI) was provided under USDA-ARS

266 Material Transfer Research Agreement 58-8042-9-0010F. Access to whole-genome sequence

267 data from the 1000 Bull Genomes Project was provided under USDA-ARS Material Transfer

268 Agreement 14358. Authors also thank Bethany Redel and Joshua Benne from the University of

269 Missouri for their assistance with microinjections of bovine embryos. Mention of trade names or 
commercial products in this article is solely for the purpose of providing specific information and

271 does not imply recommendation or endorsement by the US Department of Agriculture. The

272 USDA is an equal opportunity provider and employer.

\section{Author Contributions}

274 Cole and Ortega conceived the project. Null and Hutchison identified and annotated variant sites

275 within the haplotype region. Ortega and Clark designed CRISPR-Cas9 gRNAs, generated edited

276 embryos, and performed analysis on resulting embryos. Null and Bickhart ran analysis on

277 predicted variant sites and prepared figures for publication. McClure assisted with image

278 preparation. Ortega and Bickhart wrote the manuscript. All authors read and edited the

279 manuscript.

\section{References}

281 1. Cole, J. B., Null, D. J. \& VanRaden, P. M. Phenotypic and genetic effects of recessive

282 haplotypes on yield, longevity, and fertility. J. Dairy Sci. 99, 7274-7288 (2016).

283 2. Cole, J. B. et al. AIP Research Report Genomic5: Haplotype tests for economically

284 important traits of dairy cattle (update).

285 https://www.ars.usda.gov/ARSUserFiles/80420530/Publications/ARR/Haplotype\%20tests_A RR-Genomic5.pdf (2020).

287 3. VanRaden, P. M., Olson, K. M., Null, D. J. \& Hutchison, J. L. Harmful recessive effects on 288 fertility detected by absence of homozygous haplotypes. J. Dairy Sci. 94, 6153-6161 (2011).

289 4. Rosen, B. D. et al. De novo assembly of the cattle reference genome with single-molecule 290 sequencing. Gigascience 9, (2020).

291 5. Zimin, A. V. et al. A whole-genome assembly of the domestic cow, Bos taurus. Genome Biol. 10, R42 (2009). 
293 6. VanRaden, P. M. et al. Genomic imputation and evaluation using high-density Holstein genotypes. J. Dairy Sci. 96, 668-678 (2013).

295

7. Hayes, B. J. \& Daetwyler, H. D. 1000 Bull Genomes Project to map simple and complex genetic traits in cattle: Applications and outcomes. Ann. Rev. Anim. Biosci. 7, 89-102 (2019).

8. Huang, D. W., Sherman, B. T. \& Lempicki, R. A. Bioinformatics enrichment tools: paths toward the comprehensive functional analysis of large gene lists. Nucleic Acids Res. 37, 1-13

299 (2009).

9. Beales, P. L. et al. IFT80, which encodes a conserved intraflagellar transport protein, is mutated in Jeune asphyxiating thoracic dystrophy. Nat. Genet. 39, 727-729 (2007).

10. Rix, S., Calmont, A., Scambler, P. J. \& Beales, P. L. An Ift80 mouse model of short rib polydactyly syndromes shows defects in hedgehog signalling without loss or malformation of cilia. Hum. Mol. Genet. 20, 1306-1314 (2011).

11. Graf, A. et al. Fine mapping of genome activation in bovine embryos by RNA sequencing. Proc. Natl. Acad. Sci. USA 111, 4139-4144 (2014).

12. Yuan, X., Cao, X. \& Yang, S. IFT80 is required for stem cell proliferation, differentiation, and odontoblast polarization during tooth development. Cell Death \& Disease 10, 1-11 (2019). 
315 15. VanRaden, Paul M., O’Connell, Jeffrey R., Wiggans, G. R. \& Weigel, K. A. Genomic evaluations with many more genotypes. Genet. Sel. Evol. 43, 10 (2011).

16. Li, H. Aligning sequence reads, clone sequences and assembly contigs with BWA-MEM. arXiv:1303.3997 [q-bio] (2013).

17. Li, H. et al. The Sequence Alignment/Map format and SAMtools. Bioinformatics 25, 20782079 (2009).

18. McKenna, A. et al. The Genome Analysis Toolkit: a MapReduce framework for analyzing next-generation DNA sequencing data. Genome Res. 20, 1297-1303 (2010). (2021).

20. Cingolani, P. et al. A program for annotating and predicting the effects of single nucleotide polymorphisms, SnpEff: SNPs in the genome of Drosophila melanogaster strain w1118; iso2; iso-3. Fly (Austin) 6, 80-92 (2012).

21. Ortega, M. S. et al. Influences of sire conception rate on pregnancy establishment in dairy cattle. Biol. Reprod. 99, 1244-1254 (2018).

22. Ortega, M. S. et al. NANOG is required to form the epiblast and maintain pluripotency in the bovine embryo. Mol. Reprod. Devel. 87, 152-160 (2019). Production and Culture of the Bovine Embryo. in Comparative Embryo Culture (ed. Herrick, J. R.) vol. 2006 115-129 (Springer New York, 2019). associated with embryonic development in the cow: Evidence for a role for WBP1 in development to the blastocyst stage. PLOS ONE 12, e0178041 (2017). 

context, the IFT80 gene is drawn with exons (straight lines) and introns (diagonal lines) above

347 the concordance plot. Only variants predicted to have high functional effect by SNPEff are highlighted by labels showing coordinate numbers.
25. Goossens, K. et al. Selection of reference genes for quantitative real-time PCR in bovine preimplantation embryos. BMC Devel. Biol. 5, 27 (2005).

\section{Figure Legends}

Figure 1: A UCSC genome browser plot (a) shows the locations of prior Holstein Haplotype 2 (HH2) loci as determined in December 2013, April 2020 and August 2020. The IFT80 gene is highlighted by red dashed lines. Concordance analysis of variant sites against expected carrier status (b) identified only one variant with $>99 \%$ concordance (highlighted in red). For genomic

Figure 2: A diagram of the IFT80 gene (a) shows CRISPR-Cas9 edit sites (red triangles) and

351 qPCR primer design sites (blue boxes). Gene exons are represented by vertical lines, whereas

352 introns are represented by diagonal lines. Embryo counts for each developmental stage for exon

3532 (b) and exon 3 (c) demonstrate that homozygous edit embryos (red) do not typically progress

354 past the 8-cell stage compared to vector Cas9 controls (blue). Single $(*)$ and double $(* *)$

355 asterisks indicate Student's T test p-values that are less than 0.001 and 0.0001 , respectively.

356 Relative fold change of exons downstream of the exon 2 (d) and exon 11 (e) edit sites showed

357 significant decreases in edited embryos (red) compared to the vector Cas9 controls (blue). 
bioRxiv preprint doi: https://doi.org/10.1101/2021.07.02.450952; this version posted July 3, 2021. The copyright holder for this preprint (which

Figure 1

a
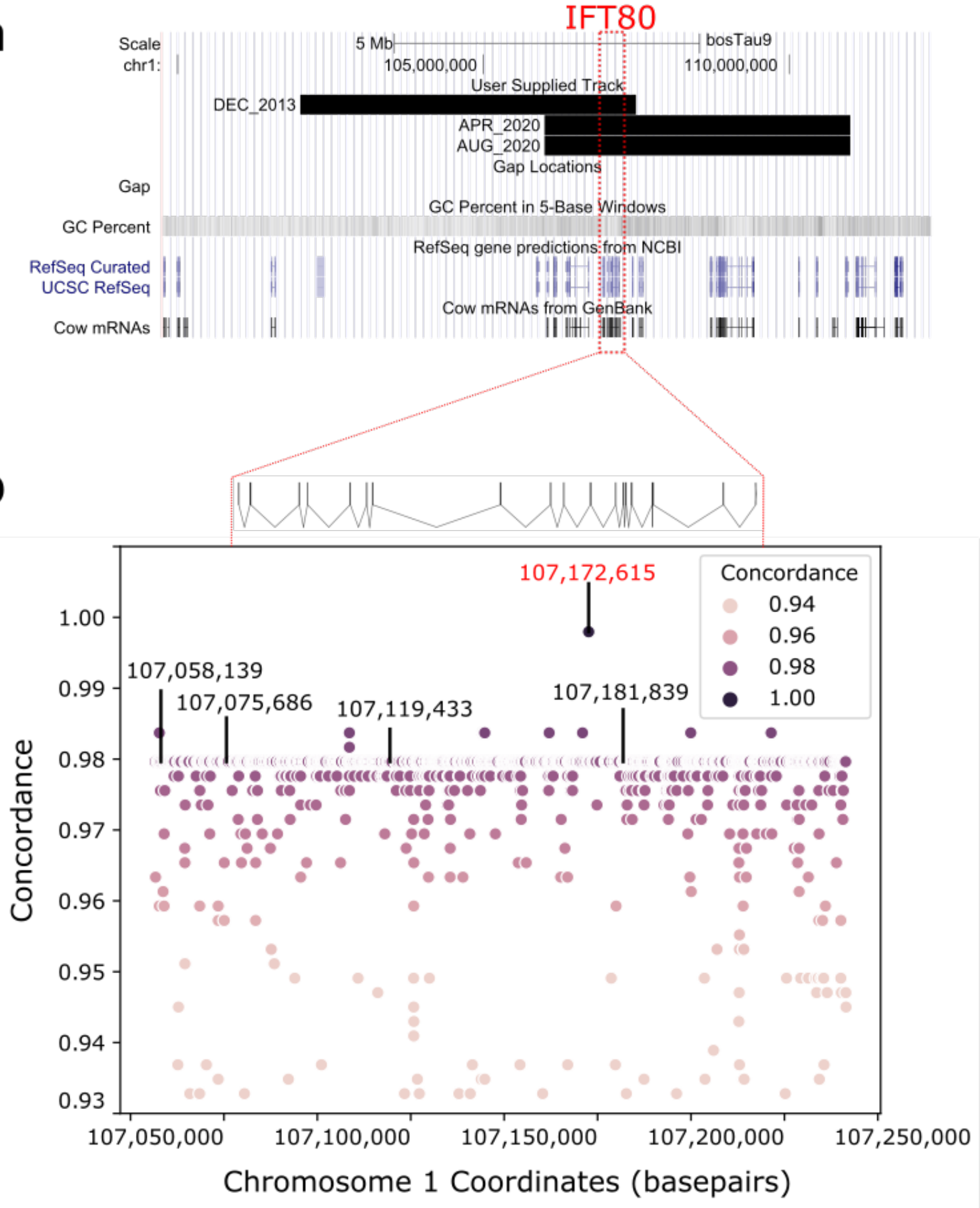
bioRxiv preprint doi: https://doi.org/10.1101/2021.07.02.450952; this version posted July 3, 2021. The copyright holder for this preprint (which was not certified by peer review) is the author/funder. This article is a US Government work. It is not subject to copyright under 17 USC 105 and is also made available for use under a CCO license.

Figure 2

a
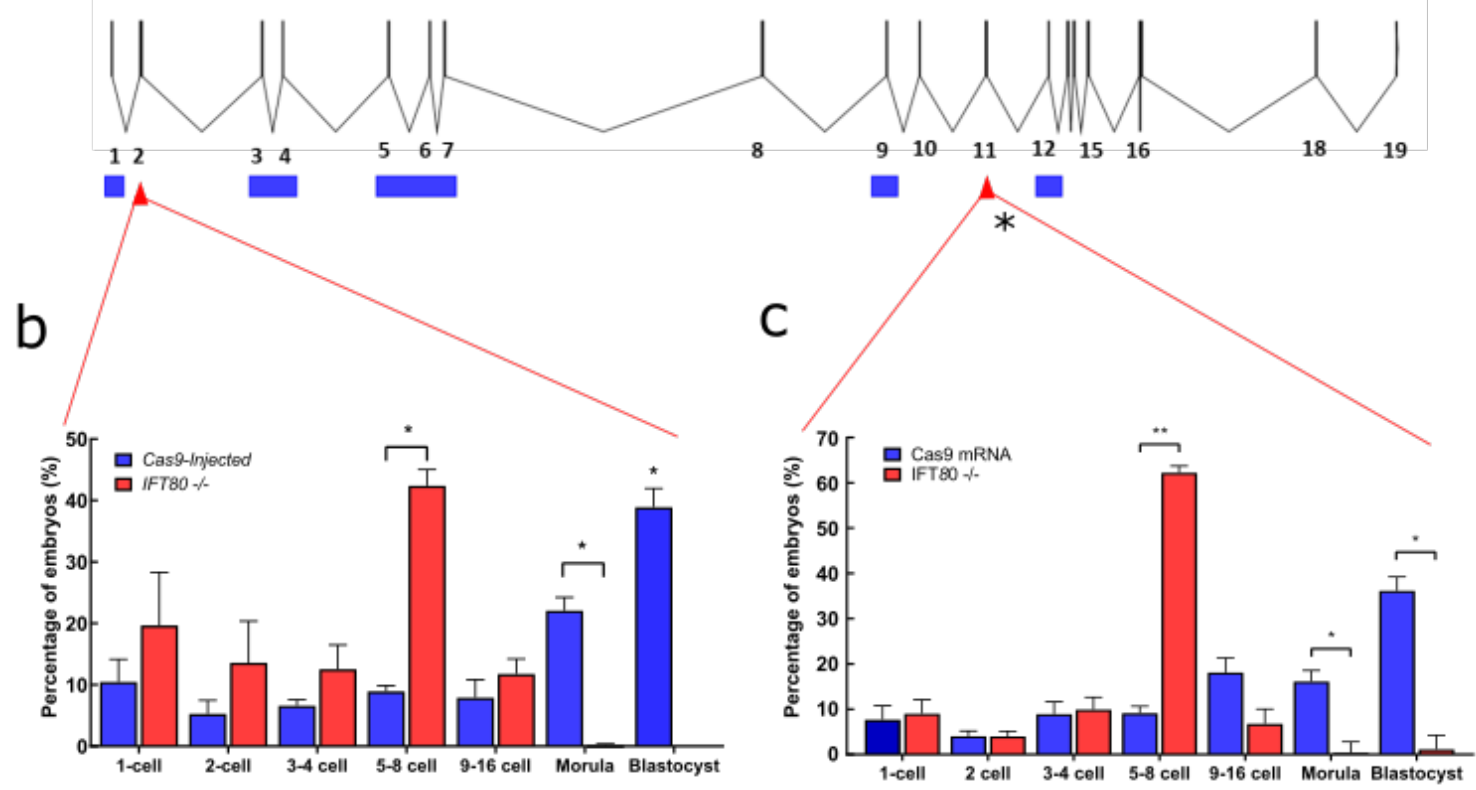

d

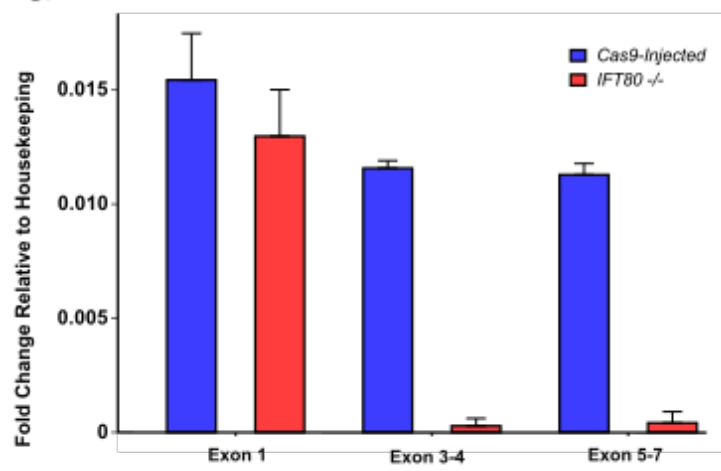

e

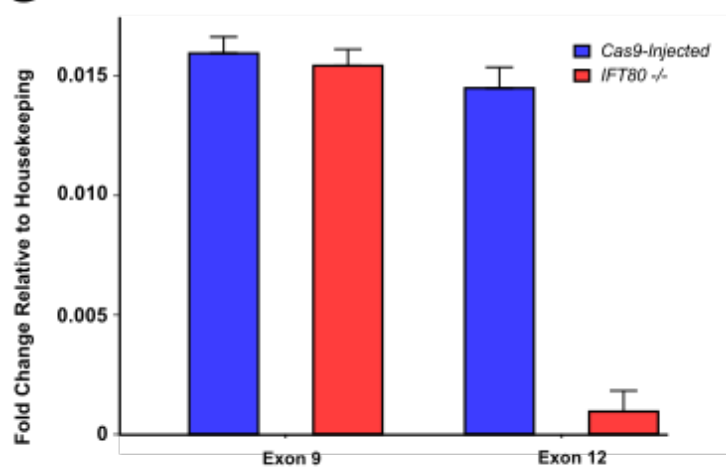

